\title{
Free Energy Functional for Nonequilibrium Systems : An Exactly Solvable Case
}

\author{
B. Derrida ${ }^{\dagger}$, J. L. Lebowitz ${ }^{\ddagger *}$ and E. R. Speer ${ }^{\ddagger}$ \\ $\dagger$ Laboratoire de Physique Statistique, 24 rue Lhomond, 75231 Paris Cedex 05, France; \\ $\ddagger$ Department of Mathematics, Rutgers University, New Brunswick, NJ 08903; \\ * Also Department of Physics, Rutgers University, New Brunswick, NJ 08903; \\ emails: derrida@lps.ens.fr, lebowitz@math.rutgers.edu,speer@math.rutgers.edu
}

\begin{abstract}
We consider the steady state of an open system in which there is a flux of matter between two reservoirs at different chemical potentials. For a large system of size $N$, the probability of any macroscopic density profile $\rho(x)$ is $\exp [-N \mathcal{F}(\{\rho\})] ; \mathcal{F}$ thus generalizes to nonequilibrium systems the notion of free energy density for equilibrium systems. Our exact expression for $\mathcal{F}$ is a nonlocal functional of $\rho$, which yields the macroscopically long range correlations in the nonequilibrium steady state previously predicted by fluctuating hydrodynamics and observed experimentally. PACS:02.50.+s, 05.40.+j, 05.70 Ln, 82.20-w
\end{abstract}

The extension of the central object of equilibrium statistical mechanics, entropy or free energy, to nonequilibrium systems in which there is a transport of matter or energy has been the holy grail of nonequilibrium statistical mechanics since the time of Boltzmann. An important step in that direction was taken by Onsager and Machlup [1] for linear deviations from equilibrium, and there have been many further extensions to time dependent evolutions starting and remaining in local equilibrium [2 [6]. The extension to stationary nonequilibrium states, for which one has no a priori control of how close the system is to local equilibrium, has however remained difficult. One knows from approximate theories like fluctuating hydrodynamics [7] that such states exhibit long range correlations totally absent from equilibrium systems (even at the critical point). These correlations have been measured experimentally in a fluid with a steady heat current [8]. Their derivation from a well defined macroscopic functional valid beyond local equilibrium is clearly an essential step in understanding pattern formation in more general stationary nonequilibrium states such as the Bénard system. We report here what we believe is the first exact derivation of such a functional for a nonequilibrium model which is relatively simple but exhibits the realistic feature of macroscopically long range correlations.

For an equilibrium system, such as a lattice gas, in a unit cube containing $L^{d}$ sites with spacing $1 / L$ (or a similar continuum system), at temperature $T$ and chemical potential $\nu$, the probability of observing a specified macroscopic density profile, with density $\rho(x)$ at macroscopic position $x$ in the unit cube, is given by $P(\{\rho(x)\}) \sim \exp \left[-L^{d} \mathcal{F}_{\text {eq }}(\{\rho\})\right]$. Here $\mathcal{F}_{\text {eq }}(\{\rho\})=$ $\int[f(\rho(x))-f(\bar{\rho}(x))] d x$, where the integration is over the unit cube, with $f(\rho)$ the grand canonical free energy density and $\bar{\rho}$ the equilibrium density profile, obtained by minimizing $\int f(\rho(x)) d x[9]$. The profile $\bar{\rho}(x)$ will be independent of $x$ unless there is an external potential. We have suppressed the dependence of $f$ and $\mathcal{F}_{\text {eq }}$ on the constant temperature $T$, and assume that neither $\rho$ nor $\bar{\rho}$ passes through a phase transition region at this temperature.

In this letter we generalize the expression for $\mathcal{F}_{\text {eq }}$ to the case of a system maintained, by contact with two boundary reservoirs at unequal chemical potentials $\nu_{0}$ and $\nu_{1}$, in a stationary nonequilibrium state with a constant particle flux. We consider perhaps the simplest such system, the one-dimensional symmetric simple exclusion process on a lattice of $N$ sites with open boundaries [10]. Each site $i, i=1, \ldots, N$, is either empty $\left(\tau_{i}=0\right)$ or occupied by a single particle $\left(\tau_{i}=1\right)$. Each particle independently attempts to jump to its right neighboring site, and to its left neighboring site, in each case at rate 1 . It succeeds if the target site is empty; otherwise nothing happens. At the boundary sites, 1 and $N$, particles are added or removed: a particle is added to site 1 , when the site is empty, at rate $\alpha$, and removed, when the site is occupied, at rate $\gamma$; similarly particles are added to site $N$ at rate $\delta$ and removed at rate $\beta$. We can think of sites $i=0$ and $i=N+1$ as occupied with probabilities $\rho_{0}=\exp \nu_{0} /\left(1+\exp \nu_{0}\right)=\alpha /(\alpha+\gamma)$ and $\rho_{1}=\exp \nu_{1} /\left(1+\exp \nu_{1}\right)=\delta /(\beta+\delta)$, independent of $\tau$. We will assume for definiteness that $\rho_{0}>\rho_{1}$, but of course, due to the left-right symmetry, this is not a restriction.

The probabilities of the microscopic configurations in the steady state may be calculated through the so-called matrix method [11]. Here we ask for the probability of seeing a specified macroscopic density profile $\rho(x)$, where $0 \leq x \leq 1$ and $0 \leq \rho(x) \leq 1$. By definition, this is the sum of the probabilities of all microscopic configurations consistent with the given profile. We shall not give a precise definition (see [3 9.9]) of "consistent" here; roughly speaking we include all configurations $\tau$ such that for any $y, z$ with $0 \leq y<z \leq 1,\left|\frac{1}{N} \sum_{i=y N}^{z N} \tau_{i}-\int_{y}^{z} \rho(x) d x\right|<$ $\delta_{N}$, with $\delta_{N} \rightarrow 0$ as $N \rightarrow \infty$.

Our main result is a parametric formula for this probability: for large $N, P(\{\rho(x)\}) \sim \exp [-N \mathcal{F}(\{\rho\})]$, with 


$$
\begin{aligned}
\mathcal{F}(\{\rho\}) & \equiv \int_{0}^{1} d x\left\{\rho(x) \log \left(\frac{\rho(x)}{F(x)}\right)\right. \\
& \left.+(1-\rho(x)) \log \left(\frac{1-\rho(x)}{1-F(x)}\right)+\log \left(\frac{F^{\prime}(x)}{\rho_{1}-\rho_{0}}\right)\right\} .
\end{aligned}
$$

Here $F$ is an auxiliary function determined by $\rho(x)$ : it is the decreasing solution of the differential equation

$$
\rho(x)=F(x)+\frac{F(x)[1-F(x)] F^{\prime \prime}(x)}{F^{\prime}(x)^{2}},
$$

satisfying the boundary conditions

$$
F(0)=\rho_{0}, \quad F(1)=\rho_{1} .
$$

It can be shown that such a solution exists and is (at least when $\rho_{1}>0$ and $\left.\rho_{0}<1\right)$ unique [15].

We note that if one looks for a monotone function $F$, satisfying the constraint (3), for which the expression $\mathcal{G}(\{\rho\},\{F\})$ given by the right hand side of (1]) is stationary, one obtains (2) as an Euler-Lagrange equation:

$$
\frac{\delta \mathcal{G}(\{\rho\},\{F\})}{\delta F(x)}=0 .
$$

We can in fact prove that this stationary point is a maximum 15] when $0<\rho_{1}<\rho_{0}<1$ and expect this to be true in general:

$$
\mathcal{F}(\{\rho\})=\sup _{F} \mathcal{G}(\{\rho\},\{F\}) .
$$

Before explaining the main steps of the derivation of (143), let us comment some of their consequences:

(a) By its very nature $\mathcal{F}$ satisfies $\mathcal{F}(\{\rho\}) \geq 0$, with equality only for $\rho(x)=\bar{\rho}(x)$, where $\bar{\rho}(x)=\rho_{0}(1-x)+$ $\rho_{1} x$ is the profile obtained with probability one in the limit $N \rightarrow \infty$. Any other profile will have $\mathcal{F}(\{\rho\})>0$ and thus, for large $N$, exponentially small probability. When $\rho_{0}=1$ or $\rho_{1}=0$ there are some profiles for which $\mathcal{F}=+\infty$; their probability is super-exponentially small in $N$. For examples, see (b) below and [15].

(b) For a constant profile $\rho(x)=r, F$ satisfies $F^{\prime}=$ $A F^{r}(1-F)^{1-r}$, where $A$ is fixed by (3), and

$$
\mathcal{F}(\{\rho\})=\log \left[\int_{\rho_{0}}^{\rho_{1}}\left(\frac{r}{z}\right)^{r}\left(\frac{1-r}{1-z}\right)^{1-r} \frac{d z}{\rho_{1}-\rho_{0}}\right] .
$$

We see that $\mathcal{F}(\{\rho\})=\infty$ if $r=1$ and $\rho_{1}=0$, or $r=0$ and $\rho_{0}=1$.

(c) Using (4), one finds immediately that

$$
\frac{\delta \mathcal{F}}{\delta \rho(x)}=\log \left[\frac{\rho(x)}{F(x)} \cdot \frac{1-F(x)}{1-\rho(x)}\right] .
$$

This expression can then be used to find optimal profiles subject to various constraints. For example, setting (7) to zero implies that $\rho(x)=F(x)$, leading to the most likely profile $\bar{\rho}(x)$ given in (a). (d) If we minimize $\mathcal{F}$ subject to the constraint of a fixed mean density $\int_{0}^{1} \rho(x) d x$, the right hand side of (7) becomes an arbitrary constant, and together with (2) one obtains that the most likely profile is exponential: $\rho(x)=A_{1} \exp (\theta x)+A_{2}$, the constants being determined by the value of the mean density and the boundary conditions (3). (This exponential form, which is the stationary solution of a diffusion equation with drift, was first suggested to us, for some special cases, by Errico Presutti).

Similarly, if we impose a fixed mean density in $k$ nonoverlapping intervals, with no other constraints, the optimal profile is exponential inside these intervals, linear outside, and in general not continuous at the end points of the intervals.

(e) When the chemical potentials of the two reservoirs are equal, i.e., $\rho_{0}=\rho_{1}$, the system is in true equilibrium with $\bar{\rho}(x)=\rho_{1}$. Eqs. (1-3) have a well-defined limit for $\rho_{1} \nearrow \rho_{0}$, with $F(x)=\rho_{0}+\left(\rho_{1}-\rho_{0}\right) x+O\left(\left(\rho_{1}-\rho_{0}\right)^{2}\right)$. It is also natural to consider a local equilibrium Gibbs measure corresponding to a spatially varying chemical potential [2,9] which is adjusted to maintain the same optimal profile $\bar{\rho}(x)$. For this system the large deviation functional (free energy) is just $\mathcal{F}_{\text {eq }}(\{\rho\})$, which has the explicit form

$$
\begin{aligned}
\mathcal{F}_{\text {eq }}(\{\rho\})=\int\{ & \rho(x) \log \frac{\rho(x)}{\bar{\rho}(x)} \\
& \left.+[1-\rho(x)] \log \frac{(1-\rho(x))}{(1-\bar{\rho}(x))}\right\} d x .
\end{aligned}
$$

From (5): $\mathcal{F}(\{\rho\}) \geq \mathcal{G}(\{\rho\},\{\bar{\rho}\})=\mathcal{F}_{\text {eq }}(\{\rho\})$, so that expressions (11) and (8) are in general different

$$
\mathcal{F}(\{\rho\}) \geq \mathcal{F}_{\text {eq }}(\{\rho\}),
$$

with equality only for $\rho(x)=\bar{\rho}(x)$ or $\rho_{0}=\rho_{1}$;

(f) Using the fact that the exponential is the optimal profile for the case of a fixed mean density in the entire interval, we may compute the distribution of $M$, the total number of particles in the system, in the steady state for large $N$. We find that the fluctuations of $M$ predicted by (1) are reduced in comparison to those in a system in local equilibrium (8) with the same $\bar{\rho}$ :

$$
\begin{aligned}
\lim _{N \rightarrow \infty} N^{-1}\left[\left\langle M^{2}\right\rangle_{\mathrm{SNS}}-\langle M\rangle^{2}\right] \\
\quad=\lim _{N \rightarrow \infty} N^{-1}\left[\left\langle M^{2}\right\rangle_{\mathrm{eq}}-\langle M\rangle^{2}\right]-\frac{\left(\rho_{1}-\rho_{0}\right)^{2}}{12} .
\end{aligned}
$$

We may also obtain (10) by expanding $\rho(x)$ about $\bar{\rho}(x)$ in (1). The result agrees with that obtained in [14] directly from the microscopic model and from fluctuating hydrodynamics [7].

Derivation: let us now sketch the derivation of (11-3). The probability of a configuration $\tau=\left\{\tau_{1}, \ldots, \tau_{N}\right\}$ in the steady state of our model is given by 11]

$$
P_{N}(\tau)=\frac{\omega_{N}(\tau)}{\left\langle W\left|(D+E)^{N}\right| V\right\rangle},
$$


where the weights $\omega_{N}(\tau)$ are given by

$$
\omega_{N}(\tau)=\left\langle W\left|\Pi_{i=1}^{N}\left(\tau_{i} D+\left(1-\tau_{i}\right) E\right)\right| V\right\rangle
$$

and the matrices $D$ and $E$ and the vectors $|V\rangle$ and $\langle W|$ satisfy

$$
\begin{gathered}
D E-E D=D+E, \\
(\beta D-\delta E)|V\rangle=|V\rangle, \quad\langle W|(\alpha E-\gamma D)=\langle W| .
\end{gathered}
$$

Although proving that (11-14) do give the weights in the steady state is rather easy [11], there is not so far a simple physical interpretation of the matrices $D$ and $E$ or of the vectors $|V\rangle$ or $\langle W|$.

To obtain the probability $P_{N_{1}, \ldots, N_{n}}\left(M_{1}, M_{2}, \ldots, M_{n}\right)$ that $M_{1}$ particles are located on the first $N_{1}$ sites, $M_{2}$ particles on the next $N_{2}$ sites, etc.., we first calculate the sum $\Omega_{N_{1}, \ldots, N_{n}}\left(M_{1}, M_{2}, \ldots, M_{n}\right)$ of the weights of all the corresponding configurations. The key to obtaining (11) is that the following generating function, which plays the role of the grand-canonical-pressure partition function, can be computed exactly:

$$
\begin{aligned}
& Z\left(\lambda_{1}, \ldots, \lambda_{n} ; \mu_{1}, \ldots, \mu_{n}\right) \\
& \quad \equiv \sum \frac{\mu_{1}^{N_{1}}}{N_{1} !} \cdots \frac{\mu_{n}^{N_{n}}}{N_{n} !} \lambda_{1}^{M_{1}} \cdots \lambda_{n}^{M_{n}} \frac{\Omega_{N_{1}, \ldots, N_{n}}\left(M_{1}, \ldots, M_{n}\right)}{\langle W \mid V\rangle} \\
& =\frac{\left\langle W\left|e^{\mu_{1} \lambda_{1} D+\mu_{1} E} \cdots e^{\mu_{n} \lambda_{n} D+\mu_{n} E}\right| V\right\rangle}{\langle W \mid V\rangle}
\end{aligned}
$$

where the sum is over all $N_{i}, M_{i} \geq 0$ and the parameters $\mu_{i}$ and $\lambda_{i}$ are conjugate to the $N_{i}$ and $M_{i}$. To do the calculation we used repeatedly the following identity, which follows from (13),

$$
e^{x D+y E}=\left(\frac{(x-y) e^{y}}{x e^{y}-y e^{x}}\right)^{E}\left(\frac{(x-y) e^{x}}{x e^{y}-y e^{x}}\right)^{D},
$$

and the result is that

$$
Z=\left(\frac{\rho_{0}-\rho_{1}}{g}\right)^{a+b} \exp \left[a \sum_{i=1}^{n} \mu_{i}\left(1-\lambda_{i}\right)\right],
$$

where $a=(\alpha+\gamma)^{-1}, b=(\beta+\delta)^{-1}$, and

$$
\begin{aligned}
g= & {\left[-\rho_{1}+\rho_{0} e^{\sum_{i=1}^{n} \mu_{i}\left(1-\lambda_{i}\right)}\right.} \\
& \left.+\sum_{i=1}^{n} \frac{1}{\lambda_{i}-1}\left(e^{\mu_{i}\left(1-\lambda_{i}\right)}-1\right) e^{\sum_{j>i} \mu_{j}\left(1-\lambda_{j}\right)}\right],
\end{aligned}
$$

All the rest of the derivation consists in extracting from the exact expression (17) the behavior of $\Omega$ and $P$ for large $N_{i}$ 's.

First (17) gives the normalization factor in (11),

$$
\frac{\left\langle W\left|(D+E)^{N}\right| V\right\rangle}{\langle W \mid V\rangle}=\frac{\Gamma(a+b+N)}{\Gamma(a+b)\left(\rho_{0}-\rho_{1}\right)^{N}},
$$

so that $P_{N_{1}, \ldots, N_{n}}\left(M_{1}, M_{2}, \ldots, M_{n}\right)$ is given simply by $\Omega_{N_{1}, \ldots, N_{n}}\left(M_{1}, M_{2}, \ldots, M_{n}\right)$ divided by (19).

Now the large $N_{i}$ behavior of $\Omega_{N_{1}, \ldots, N_{n}}$ controls the position and the nature of the singularities of $Z$ closest to the origin $\mu_{i}=\lambda_{i}=0$; conversely, this relation can be inverted [15] to determine the asymptotic behavior of $\Omega_{N_{1}, \ldots, N_{n}}$ from the equation $g=0$ of the surface on which $Z$ is singular, just as the growth of the coefficients of a power series of one variable is determined by the singularity nearest the origin. In particular, if one sets $N_{j}=N y_{j}$ and $M_{j}=N_{j} r_{j}$ then, for large $N$ and fixed $y_{i}, r_{i}$,

$$
\begin{aligned}
& \frac{\log P_{N_{1}, \ldots, N_{n}}\left(M_{1}, \ldots, M_{n}\right)}{N} \\
& \simeq \log \left(\rho_{0}-\rho_{1}\right)-\sum_{j=1}^{n} y_{j}\left(\log \mu_{j} / y_{j}+r_{j} \log \lambda_{j}\right),
\end{aligned}
$$

where $y_{j}$ and $r_{j}$ are related to the parameters $\mu_{1}, \ldots, \mu_{n}$ and $\lambda_{1}, \ldots, \lambda_{n}$ by

$$
y_{j}=\frac{\frac{\partial g}{\partial \log \mu_{j}}}{\sum_{i=1}^{n} \frac{\partial g}{\partial \log \mu_{i}}}, \quad r_{j}=\frac{\frac{\partial g}{\partial \log \lambda_{j}}}{\frac{\partial g}{\partial \log \mu_{j}}},
$$

with all derivatives calculated on the manifold $g=0$.

Equations (20) and (21) determine $\mathcal{F}$ in a parametric form. As the $\mu_{j}$ 's and the $\lambda_{j}$ 's vary, they give the sizes of the boxes $y_{j}$, their particle densities $r_{j}$, and the corresponding probabilities. Note that the parameters $a$ and $b$ do not appear in (18) and therefore in the equation $g=0$; so for large $N$, only $\rho_{0}$ and $\rho_{1}$ remain relevant.

This parametric form can be simplified by replacing the role of the $\mu_{i}$ and $\lambda_{i}$ by a single sequence of parameters $G_{i}$. Let us define the constant $C$ by $C=$ $\sum_{i=1}^{n} \partial g / \partial \log \mu_{i}$ and the sequence $G_{i}$ by

$$
G_{i}=C^{-1} e^{\sum_{j=i}^{n} \mu_{j}\left(1-\lambda_{j}\right)}, \quad G_{n+1}=C^{-1} .
$$

It follows from (22) that $\mu_{i}=\log \left(G_{i} / G_{i+1}\right) /\left(1-\lambda_{i}\right)$ and

$$
\begin{aligned}
\frac{1}{\lambda_{i}-1}=\frac{1}{G_{i+1}} & \frac{y_{i}}{\log \left(G_{i} / G_{i+1}\right)}-\rho_{0} \\
& +\sum_{j=1}^{i}\left(\frac{1}{G_{j}}-\frac{1}{G_{j+1}}\right) \frac{y_{j}}{\log \left(G_{j} / G_{j+1}\right)} .
\end{aligned}
$$

The condition that $g=0$ becomes

$$
\rho_{0}-\rho_{1}=\sum_{j=1}^{n}\left(\frac{1}{G_{j}}-\frac{1}{G_{j+1}}\right) \frac{y_{j}}{\log \left(G_{j} / G_{j+1}\right)},
$$

which can be thought of as an equation which determines $G_{n+1}$ in terms of $G_{1}, \ldots, G_{n}$.

Once the $\lambda_{i}$ 's are known, one gets for the $r_{i}$ 's and the large deviation function 


$$
r_{i}=-\frac{\lambda_{i}}{1-\lambda_{i}}-\frac{\lambda_{i}}{\left(1-\lambda_{i}\right)^{2}} \frac{G_{i}-G_{i+1}}{y_{i}}
$$

and

$$
\begin{aligned}
& \frac{\log \left[P_{N_{1}, \ldots, N_{n}}\left(M_{1}, \ldots, M_{n}\right)\right]}{N} \\
& =-\sum_{i=1}^{n}\left\{y_{i} \log \left[\frac{\log \left(\frac{G_{i}}{G_{i+l}}\right)}{y_{i}\left(\rho_{0}-\rho_{1}\right)}\right]\right. \\
& \left.\quad-y_{i} \log \left(1-\lambda_{i}\right)+y_{i} r_{i} \log \left(\lambda_{i}\right)\right\} .
\end{aligned}
$$

To take the limit $N \rightarrow \infty$ followed by $n \rightarrow \infty, y_{i} \rightarrow 0$, we introduce a continuous variable $x, 0 \leq x \leq 1$, and let $x_{i}=y_{1}+y_{2}+\ldots+y_{i}$. All the discrete sequences can now be thought of as functions of $x_{1}, \ldots, x_{n}$ with $G_{i} \equiv G\left(x_{i}\right), \lambda_{i} \equiv \lambda\left(x_{i}\right)$ and $r_{i} \equiv \rho\left(x_{i}\right), i=1, \ldots, n$. Then, extending $G, \lambda$, and $\rho$ to be smooth functions of $x$, so that $G_{i+1}-G_{i} \simeq y_{i} G^{\prime}\left(x_{i}\right)$, one finds that (23,24) become

$$
\begin{aligned}
\frac{1}{\lambda(x)-1} & =\frac{-1}{G^{\prime}(x)}-\rho_{0}-\int_{0}^{x} \frac{d u}{G(u)}, \\
\rho_{1} & =\rho_{0}+\int_{0}^{1} \frac{d u}{G(u)} .
\end{aligned}
$$

At this stage it is more convenient to replace $G(x)$ by the function $F(x)$ defined by $F(x)=\rho_{0}+\int_{0}^{x} G(u)^{-1} d u$. The expression (27) for $\lambda(x)$ becomes then

$$
\frac{1}{\lambda(x)-1}=\frac{F^{\prime}(x)^{2}}{F^{\prime \prime}(x)}-F(x)
$$

Using the above relations we may rewrite (25) and (26) in terms of $F$, obtaining (11) and (2); the boundary conditions (3) come from (28). The monotonicity of $F$ follows from the uniqueness of the sign of $G$ (see (22)).

Possible extensions: It would be nice to give a physical interpretation of the Euler-Lagrange equations (4, 5i) directly from a macroscopic or semimacroscopic approach. Such an approach might allow an extension to the large deviation function for time-dependent profiles and permit one to unify various results recently obtained for the large deviation functions of time dependent profiles or currents [6, 12,13].

The matrix method (with modified rules $(13414)$ ) also applies 11] to the asymmetric simple exclusion process, in which particles jump to the left and right at different rates. We are at present trying to generalize our calculations to this case. We have already calculated the probability of a specified mean density $\rho$ (total number of particles) for the special case in which particles jump only to their right and with only input, with rate $\alpha>1$, at the left and output, with rate $\beta>1$, at the right:

$$
N^{-1} \log P(\rho) \sim-2[\rho \log 2 \rho+(1-\rho) \log 2(1-\rho)] .
$$

For a Bernoulli measure at uniform density $1 / 2$, the result would be the same except for the factor 2 in front of the whole expression, which here again expresses the fact that fluctuations are reduced by long range correlations.

Conclusion: For the simple model we studied here, the large deviation function $\mathcal{F}$ (which extends the notion of free energy to nonequilibrium systems) is a nonlocal functional (11-3) of the density $\rho$. This implies that the probability of a given profile is not the product of probabilities for, say, the left and right halves of the system, in contrast to the situation for equilibrium systems. Since long range correlations are expected for general stationary nonequilibrium states and have even been measured experimentally [8], the nonlocal nature of $\mathcal{F}(\{\rho\})$ is presumably also general, in contrast with the conjecture [4] that $\mathcal{F}$ is a local function of the hydrodynamic variables.

We thank E. Presutti for very helpful discussions. The work of J. L. Lebowitz was supported by NSF Grant DMR-9813268, AFOSR Grant F49620/0154, DIMACS and its supporting agencies, and NATO Grant PST.CLG.976552. J.L.L. and E. R. Speer acknowledge the hospitality of the I.H.E.S. in the spring of 2000, where this work was begun.

[1] L. Onsager, S. Machlup, Phys.Rev. 911505 (1053) ; 91 1512 (1953)

[2] C. Kipnis, S. Olla, and S. R. S. Varadhan, Commun. Pure Appl. Math. 42, 115 (1989).

[3] C. Kipnis and C. Landim, Scaling Limits of Interacting Particle Systems (Springer-Verlag, Berlin, 1999).

[4] R. Graham, in Order and Fluctuations in Equilibrium and Nonequilibrium Statistical Mechanics, ed. G. Nicolis, G. Dewel, and J. W. Turner (Wiley, New York, 1981).

[5] G. Eyink, J. Stat. Phys. 61, 533 (1990).

[6] L. Bertini et al., Phys. Rev. Lett. 87, 040601 (2001)

[7] J. R. Dorfman, T. R. Kirkpatrick, and J. V. Sengers, Annu. Rev. Phys. Chem. 45, 213 (1994).

[8] W. B. Li et al., Phys. Rev. Lett. 81, 5580 (1998).

[9] H. Spohn, Large Scale Dynamics of Interacting Particles (Springer-Verlag, Berlin, 1991).

[10] G. Eyink, J. L. Lebowitz, and H. Spohn, CMP 132, 253 (1990); 140, 119 (1991).

[11] B. Derrida, M. R. Evans, V. Hakim and V. Pasquier, J. Phys. A 26, 1493 (1993); F. H. L. Essler, V. Rittenberg J. Phys. A 29, 3375 (1996); N. Rajewsky, M. Schreckenberg Physica A 245, 139 (1997); T. Sasamoto, J. Phys. A 32, 7109 (1999); R. A. Blythe, M. R. Evans, F. Colaiori, F. H. L. Essler, J. Phys. A 33, 2313 (2000).

[12] B. Derrida and J. L. Lebowitz, Phys. Rev. Lett. 80, 209 (1998).

[13] M. Prähofer, H. Spohn, Phys. Rev. Lett. 84, 4882 (2000).

[14] H. Spohn, J. Phys A. 16, 4275 (1983).

[15] B. Derrida, J. L. Lebowitz, and E. R. Speer, in preparation. 